\title{
Young's Modulus of Polycrystalline Titania Microspheres Determined by In Situ Nanoindentation and Finite Element Modeling
}

\author{
Peida Hao, ${ }^{1,2}$ Yanping Liu, ${ }^{1}$ Yuanming Du, ${ }^{1}$ and Yuefei Zhang ${ }^{2}$ \\ ${ }^{1}$ College of Mechanical Engineering, Taiyuan University of Technology, Taiyuan 030024, China \\ ${ }^{2}$ Institute of Microstructure and Properties of Advanced Materials, Beijing University of Technology, Beijing 100022, China \\ Correspondence should be addressed to Yanping Liu; lyplr@163.com and Yuefei Zhang; yfzhang@bjut.edu.cn
}

Received 9 October 2014; Accepted 27 November 2014; Published 15 December 2014

Academic Editor: Sheng-Rui Jian

Copyright ( $\odot 2014$ Peida Hao et al. This is an open access article distributed under the Creative Commons Attribution License, which permits unrestricted use, distribution, and reproduction in any medium, provided the original work is properly cited.

In situ nanoindentation was employed to probe the mechanical properties of individual polycrystalline titania $\left(\mathrm{TiO}_{2}\right) \mathrm{microspheres}$ The force-displacement curves captured by a hybrid scanning electron microscope/scanning probe microscope (SEM/SPM) system were analyzed based on Hertz's theory of contact mechanics. However, the deformation mechanisms of the nano/microspheres in the nanoindentation tests are not very clear. Finite element simulation was employed to investigate the deformation of spheres at the nanoscale under the pressure of an AFM tip. Then a revised method for the calculation of Young's modulus of the microspheres was presented based on the deformation mechanisms of the spheres and Hertz's theory. Meanwhile, a new force-displacement curve was reproduced by finite element simulation with the new calculation, and it was compared with the curve obtained by the nanoindentation experiment. The results of the comparison show that utilization of this revised model produces more accurate results. The calculated results showed that Young's modulus of a polycrystalline $\mathrm{TiO}_{2}$ microsphere was approximately $30 \%$ larger than that of the bulk counterpart.

\section{Introduction}

Nano/microspheres have wide application in supercapacitors, biosensors, drug delivery, and catalysts [1-3]. The deformation of these nano/microspheres under an external force affects their performance. Understanding the properties of individual microspheres, such as the deformation mechanism and the mechanical properties, will aid in controlling the final structure and other interesting properties of these microspheres. Until now, experimental data on the mechanical properties of nano/microspheres are still lacking. Nano/microspheres may have properties that differ from those of bulk materials, as has been demonstrated for other types of nanomaterials [4].

The extremely small dimensions of the nano/microspheres present a formidable challenge for experimental studies of their mechanical properties. Nanoindentation is currently the only viable approach to measure the mechanical properties of nano/microspheres. Armini et al. and Chen characterized the mechanical properties of microspheres for drug delivery and polymer microspheres using the nanoindentation method $[5,6]$. In these tests, Hertz's model for contact mechanics was used to characterize the mechanical properties of the nano/microspheres by measuring the forcedisplacement curves with an AFM cantilever tip. However, these tests took the displacement of the tip as the penetration depth and neglected the compressive deformation of the spheres. An accurate penetration depth should be extracted to characterize accurately the mechanical properties.

As an important wide-bandgap semiconductor, $\mathrm{TiO}_{2}$ has been formed into nanostructured materials that have extensive applications in many fields, especially in photocatalysis, solar cells, and electrochromic devices [7]. There are a number of reports on the mechanical properties of bulk $\mathrm{TiO}_{2}$, but the existing literature addressing the mechanical properties of $\mathrm{TiO}_{2}$ microspheres is scarce. In this paper, Young's moduli of $\mathrm{TiO}_{2}$ microspheres with various radii $(300-800 \mathrm{~nm})$ were measured by performing in situ nanoindentation tests 


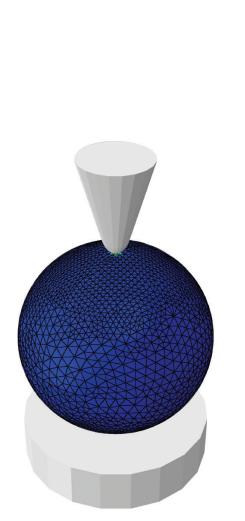

(a)

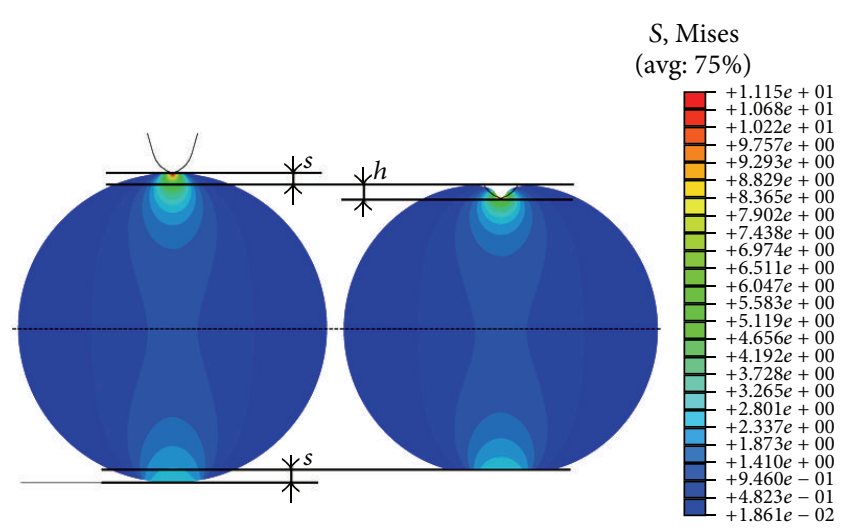

(b)

Figure 1: (a) Model of indentation of $\mathrm{TiO}_{2}$ microsphere. (b) Selected stress distribution to illustrate the deformation mechanisms. $s$ and $h$ are the compressive deformation and penetration depth of the microspheres, respectively.

using a hybrid SEM/SPM system. Moreover, finite element modeling (FEM) was used to investigate the deformation mechanism of the microspheres in the nanoindentation tests.

\section{Experimental}

The nanocrystalline $\mathrm{TiO}_{2}$ microspheres were prepared using the same process described by Crepaldi et al. [8]. Scanning electron microscopy (SEM, Quanta 250, $20 \mathrm{kV}$ ) and transmission electron microscopy (TEM, JEM-2010F, $200 \mathrm{kV}$ ) were employed to characterize the morphology and the structure of the $\mathrm{TiO}_{2}$ microspheres.

In situ nanoindentation experiments were conducted using a hybrid SEM/SPM system. The hybrid system relied on high-magnification SEM as a visual feedback system to indent the microspheres accurately with a cantilever probe. A more detailed description of the experimental setup previously can be found in the recent literature [9].

After the nanoindentation tests, FEM was used to investigate the deformation mechanisms of the nano/microspheres and to validate the calculated results. The FEM is shown in Figure 1. FEM was based on the finite element code Abaqus and consisted of three parts: a rigid substrate, a cone indenter, and a microsphere (Figure 1(a)). In the simulation, ten-node, three-dimensional tetrahedron elements were used throughout the entire model, and reduced integration was used to control the hourglass. The density of the FE mesh increases with proximity to the indenter/microsphere contact region (Figure 1(a)). The undersurface of the substrate was fixed at all directions. The contact at indenter/microsphere and microsphere/substrate interfaces was assumed to be frictionless. The microsphere was modelled as homogenous linear elastic material. The rigid indenter was considered because the AFM tip was much stiffer than the microspheres.

\section{Results and Discussion}

The morphology and structure of the $\mathrm{TiO}_{2}$ microspheres are shown in Figure 2. SEM observation reveals that the diameters of the $\mathrm{TiO}_{2}$ microspheres are in the range of $200 \mathrm{~nm}$ to $1.5 \mu \mathrm{m}$. The TEM images show that the microsphere is homogeneous and the surface is smooth and free of defects (Figure 2(b)). The XRD pattern and selected-area electron diffraction pattern indicate that the microspheres are polycrystalline.

Figure 1(b) shows the representative von Mises stress distribution for indenting a microsphere to illustrate the deformation mechanisms. The relatively large deformations are mainly concentrated at the contact points of the spheres. It agrees well with those variations of microsphere from the nanoindentation experiment. These deformations are mainly caused by the penetration of the tip and the compressive deformation of the microsphere under pressure.

To quantitatively measure Young's modulus of the $\mathrm{TiO}_{2}$ microspheres by Hertz's model, force-displacement curves should be extracted. However, the force-displacement curves produced by the hybrid SEM/SPM system were the sum of the real penetration depth $(h)$ and the compressive deformation $(s)$ of the microspheres under pressure (Figure 1(b)). The single penetration depth $(h)$ should be extracted to obtain the actual force-displacement curve.

Next, the actual force-displacement curves were fitted with the general Sneddon's expression $\left(F=\alpha h^{1.5}\right)[10]$, where $F$ is the applied force, $h$ is the penetration depth, and $\alpha$ is an unknown constant, which is calculated from a power-law fit. Reference materials were utilized to estimate the tip radius using the following:

$$
R=\left(\frac{3 \alpha_{\mathrm{ref}}}{4 E_{r(\mathrm{ref})}}\right)^{2},
$$

where $E_{r(\text { ref })}$ is the reduced elastic modulus of the reference sample (silicon wafer).

The reduced elastic modulus for the test sample $E_{r}$ was then calculated by

$$
E_{r}=\frac{3 \alpha}{4 \sqrt{R}}
$$




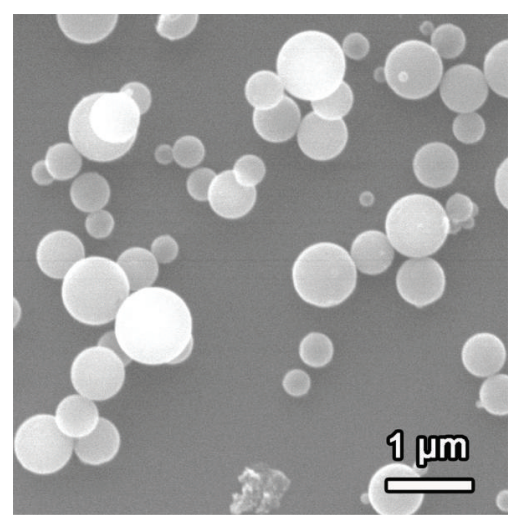

(a)

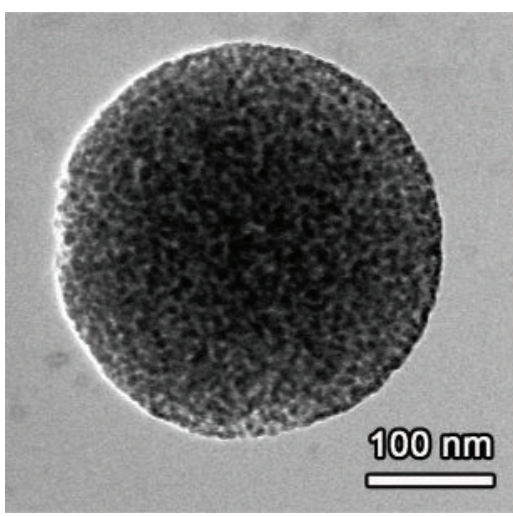

(b)

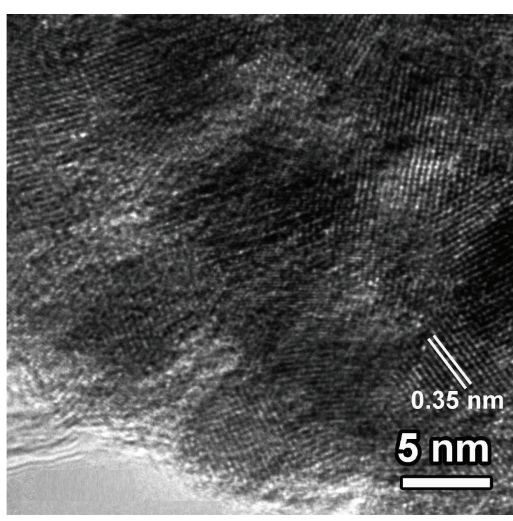

(c)

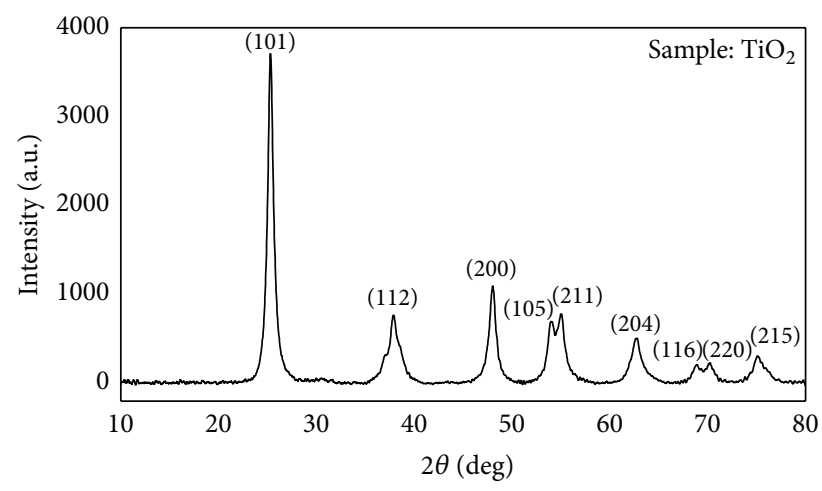

(d)

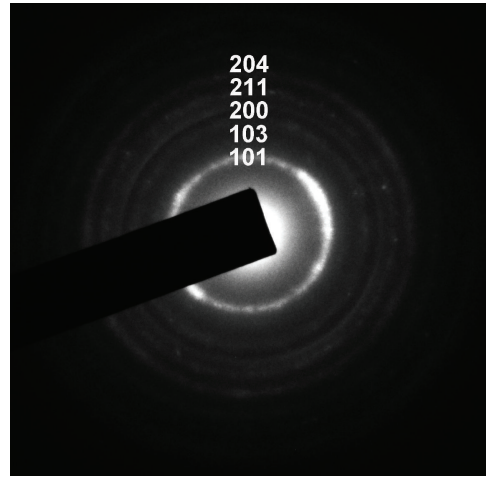

(e)

Figure 2: (a) SEM image of $\mathrm{TiO}_{2}$ microspheres. (b) TEM image of a single $\mathrm{TiO}_{2}$ microsphere. (c) HRTEM image of the microsphere shown in (b). (d) XRD patterns of $\mathrm{TiO}_{2}$ microspheres. (e) Selected-area electron diffraction (SAED) pattern recorded on the microsphere shown in (b).

Young's modulus of the sample $E_{s}$ was finally obtained using (3), where the subscript $s$ and $I$ denote the sample and tip, respectively, $v$ is Poisson's ratio, and $E$ is Young's modulus:

$$
\frac{1}{E_{r}}=\frac{1-v_{s}^{2}}{E_{s}}+\frac{1-v_{I}^{2}}{E_{I}} .
$$

The calculation procedure described above was used to obtain Young's modulus of the microspheres.

Next, the way to extract the single penetration depth (h) from the whole displacement was explored. As shown in Figure 1(b), the compressive deformations $(s)$ at the top and the bottom of the microsphere are almost identical. Therefore, we investigated the compressive deformation at the bottom of the microsphere here. Figure 3(b) shows the stress-deformation model at the bottom of the microsphere. It is similar to the model proved by Antonyuk et al. [11]. The relationship between the elastic contact force and the compressive deformation at the bottom of the microsphere is nonlinear, as described by Hertz [12]:

$$
F=\frac{2}{3} E^{*} \sqrt{r s^{3}}
$$

where $F$ is the normal force, $E^{*}$ is the effective modulus of elasticity, $r$ is the radius of the microsphere, and $s$ is the vertical deformation at the bottom of the microsphere.

The effective modulus of elasticity $E^{*}$ of both the microsphere (index 1) and the substrate (index 2) is given as

$$
E^{*}=2\left(\frac{1-v_{1}^{2}}{E_{1}}+\frac{1-v_{2}^{2}}{E_{2}}\right)^{-1} \text {. }
$$

Then the vertical deformation at the bottom of the microsphere can be converted as

$$
s=\left(\frac{3 F}{2 E^{*}}\right)^{2 / 3} \times\left(\frac{1}{r}\right)^{1 / 3} .
$$

Similarly, the penetration depth at the top of the microsphere can be converted via (2):

$$
h=\left(\frac{3 F}{4 E_{r}}\right)^{2 / 3} \times\left(\frac{1}{R}\right)^{1 / 3} .
$$

When the indenter tip and the substrate are the same material (the same material is silicon in this study), the reduced elastic modulus and the effective modulus of 


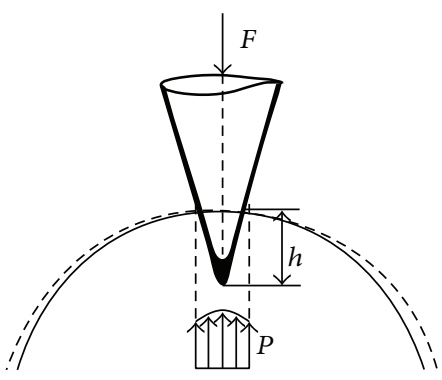

(a)

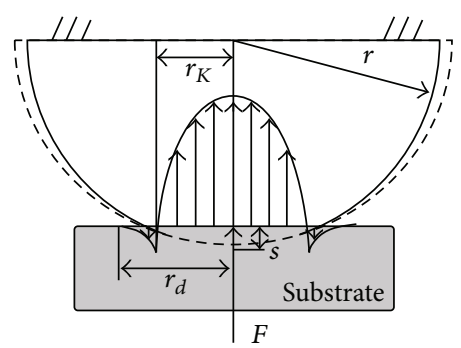

(b)

FIGURE 3: Schematic indentation of microsphere material by conical indenter. (a) Real penetration depth $(h)$ at the top of the microsphere. (b) Compressive deformation $(s)$ at the bottom of the microsphere.

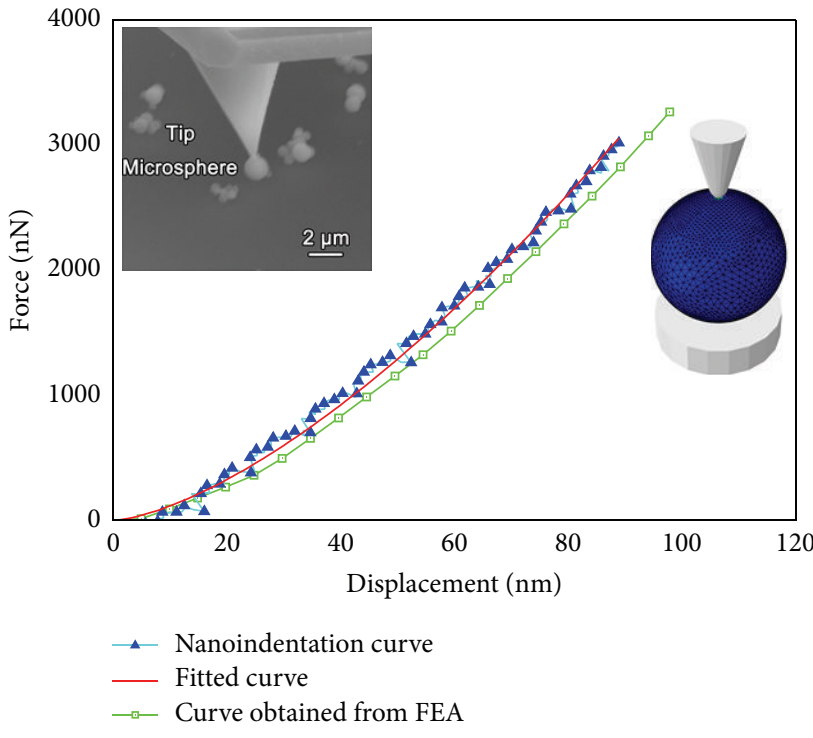

(a)

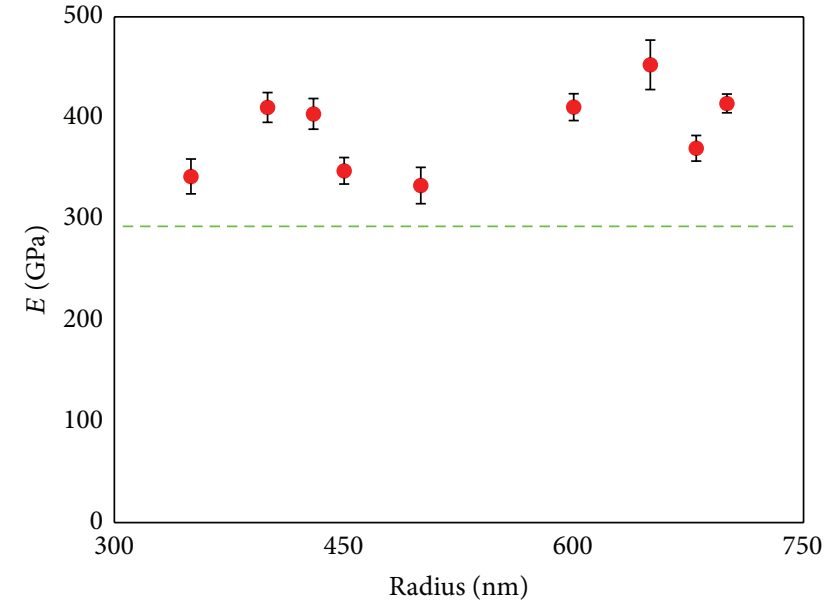

(b)

Figure 4: (a) Force-displacement curves extracted from nanoindentation experiments and finite element simulations. (b) Young's modulus as a function of microsphere diameter. The dash line represents Young's modulus of bulk titania.

elasticity have the relationship $E_{r}=E^{*} / 2$. Thus, the displacement of the indenter tip can be calculated as the sum of the penetration depth and the compressive deformation of the microsphere:

$$
H=2 s+h=\left(\frac{3 F}{2 E^{*}}\right)^{2 / 3} \times\left[\left(\frac{1}{R}\right)^{1 / 3}+\left(\frac{1}{r}\right)^{1 / 3}\right] .
$$

The force-penetration depth curve can be extracted from the force-displacement curve:

$$
h=H-2 s=\left(\frac{\sqrt[3]{1 / R}}{\sqrt[3]{1 / R}+2 \sqrt[3]{1 / r}}\right) H .
$$

After the force-penetration depth curves were obtained, Young's modulus of the $\mathrm{TiO}_{2}$ microspheres with various radii was calculated via (3) as shown in Figure 4(b). The fitting loading curve in a force-displacement curve was used to calculate Young's modulus in our study. Errors of Young's modulus measurement could come from the force and displacement measurements as well as the fitting (see error bars in Figure 4(b)). The average Young's modulus of the $\mathrm{TiO}_{2}$ microspheres is $386.1 \pm 41.8 \mathrm{GPa}$. This value is larger than that of the bulk counterpart $(282.76 \mathrm{GPa})$. This is perhaps because of these following reasons. Firstly, the defects, such as dislocations, are less as the decreasing size of the spheres. This is in agreement with the existing theories for indentation size effect that are mainly based on dislocation movement [13]. Secondly, the nonuniform density of the microsphere can be observed by TEM (Figure 2(b)). This can be considered as a core-shell structure which has no shape interface between the shell and the core. And the surface shell can play a significant role on the particle size effect [14].

Meanwhile, the results extracted from the unrevised force-displacement curve were also calculated, which show that neglecting the compressive deformation of the sphere leads to $10-15 \%$ error. FEM was used to verify the calculation results by reproducing the force-displacement curves and comparing the simulated curves with the experimental 
curves. FE models were created based on physical properties of each component in experiments. Young's modulus used in simulations was the results calculated before, while the Poisson ratio was estimated from literature [15]. As shown in Figure 4(a), the reproduced curve with Young's modulus calculated by the revised method was close to the experimental curve. This result suggests that the revised force-penetration depth curve contributes to a more accurate Young's modulus. The revised method can be used to measure Young's modulus of nano/microspheres.

\section{Conclusion}

In this study, we have used a hybrid SEM/SPM system to perform in situ nanoindentation on $\mathrm{TiO}_{2}$ microspheres, and we have investigated the deformation mechanisms for indenting a microsphere by finite element simulations. The penetration depth was extracted by removing the compressive deformation of the microsphere to obtain more accurate results. The calculated Young's modulus of the $\mathrm{TiO}_{2}$ microspheres is larger than that of their bulk counterpart as a result of size effect. Additionally, the results were verified using FEM by reproducing the force-displacement curves and comparing the simulated curves with the experimental curves. It showed that there was a good agreement between them. This work provides a useful guide for interpretation of the data obtained by indenting nano/microspheres and obtains a relatively accurate result. Further experimental investigation is necessary to verify its applicability to many other nano/microspheres.

\section{Conflict of Interests}

The authors declare that there is no conflict of interests regarding the publication of this paper.

\section{Acknowledgments}

Special Projects for Development of National Major Scientific Instruments and Equipments (2012YQ03007508), National Natural Science Fund Project (11374027), and Key Scientific and Technological Project of Shanxi Province (2013032101104) are gratefully acknowledged.

\section{References}

[1] H. Cheng, B. Huang, Z. Wang, X. Qin, X. Zhang, and Y. Dai, "One-pot miniemulsion-mediated route to $\mathrm{BiOBr}$ hollow microspheres with highly efficient photocatalytic activity," Chemistry-A European Journal, vol. 17, no. 29, pp. 8039-8043, 2011.

[2] L.-H. Hung, S.-Y. Teh, J. Jester, and A. P. Lee, "PLGA micro/nanosphere synthesis by droplet microfluidic solvent evaporation and extraction approaches," Lab on a Chip, vol. 10, no. 14, pp. 1820-1825, 2010.

[3] W. Xiong, M. Liu, L. Gan et al., "A novel synthesis of mesoporous carbon microspheres for supercapacitor electrodes," Journal of Power Sources, vol. 196, no. 23, pp. 10461-10464, 2011.
[4] X. Li, X. Wang, Q. Xiong, and P. C. Eklund, "Mechanical properties of ZnS nanobelts," Nano Letters, vol. 5, no. 10, pp. 1982-1986, 2005.

[5] S. Armini, I. U. Vakarelski, C. M. Whelan, K. Maex, and K. Higashitani, "Nanoscale indentation of polymer and composite polymer-silica core-shell submicrometer particles by atomic force microscopy," Langmuir, vol. 23, no. 4, pp. 2007-2014, 2007.

[6] J. Chen, "Understanding the nanoindentation mechanisms of a microsphere for biomedical applications," Journal of Physics D: Applied Physics, vol. 46, no. 49, Article ID 495303, 2013.

[7] J. J. Choi, X. Yang, Z. M. Norman, S. J. L. Billinge, and J. S. Owen, "Structure of methylammonium lead iodide within mesoporous titanium dioxide: active material in high-performance perovskite solar cells," Nano Letters, vol. 14, no. 1, pp. 127-133, 2014.

[8] E. L. Crepaldi, G. J. D. A. A. Soler-Illia, D. Grosso, F. Cagnol, F. Ribot, and C. Sanchez, "Controlled formation of highly organized mesoporous titania thin films: from mesostructured hybrids to mesoporous nanoanatase $\mathrm{TiO}_{2}$," Journal of the American Chemical Society, vol. 125, no. 32, pp. 9770-9786, 2003.

[9] Y. Zhang, C. Zhang, X. Han et al., 20th IEEE International Symposium on the Physical and Failure Analysis of Integrated Circuits, 2013 July 15-19, Suzhou, China, 2013.

[10] I. N. Sneddon, "The relation between load and penetration in the axisymmetric boussinesq problem for a punch of arbitrary profile," International Journal of Engineering Science, vol. 3, no. 1, pp. 47-57, 1965.

[11] S. Antonyuk, J. Tomas, S. Heinrich, and L. Mörl, "Breakage behaviour of spherical granulates by compression," Chemical Engineering Science, vol. 60, no. 14, pp. 4031-4044, 2005.

[12] H. Hertz, "Über die Berührung fester elastischer Körper," Journal für die Reine und Angewandte Mathematik, vol. 92, pp. 156-171, 1882.

[13] W. D. Nix, J. R. Greer, G. Feng, and E. T. Lilleodden, "Deformation at the nanometer and micrometer length scales: effects of strain gradients and dislocation starvation," Thin Solid Films, vol. 515, no. 6, pp. 3152-3157, 2007.

[14] J. Y. He, Z. L. Zhang, M. Midttun et al., "Size effect on mechanical properties of micron-sized PS-DVB polymer particles," Polymer, vol. 49, no. 18, pp. 3993-3999, 2008.

[15] A. V. Desai and M. A. Haque, "Mechanical properties of $\mathrm{ZnO}$ nanowires," Sensors and Actuators A: Physical, vol. 134, no. 1, pp. 169-176, 2007. 

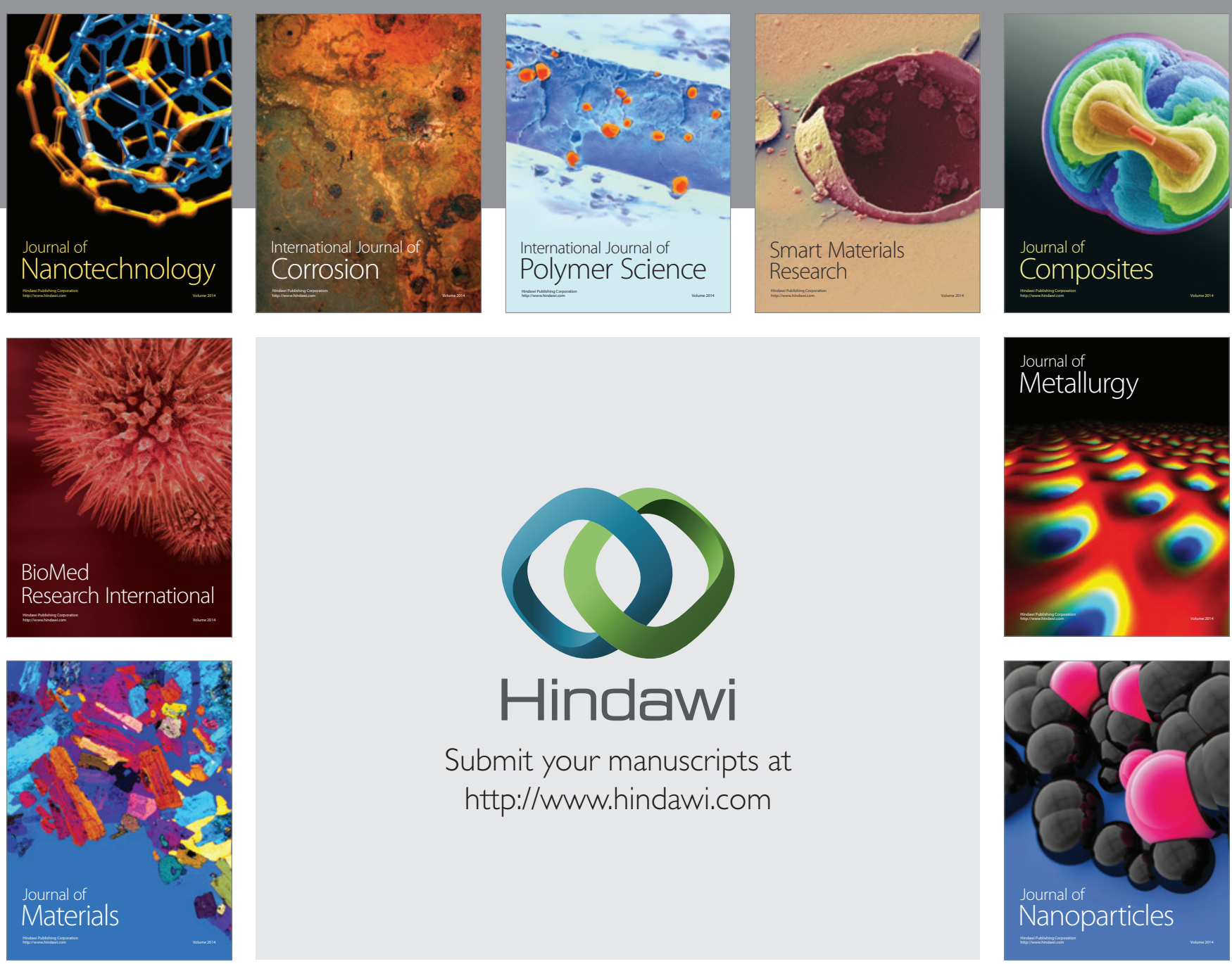

Submit your manuscripts at http://www.hindawi.com
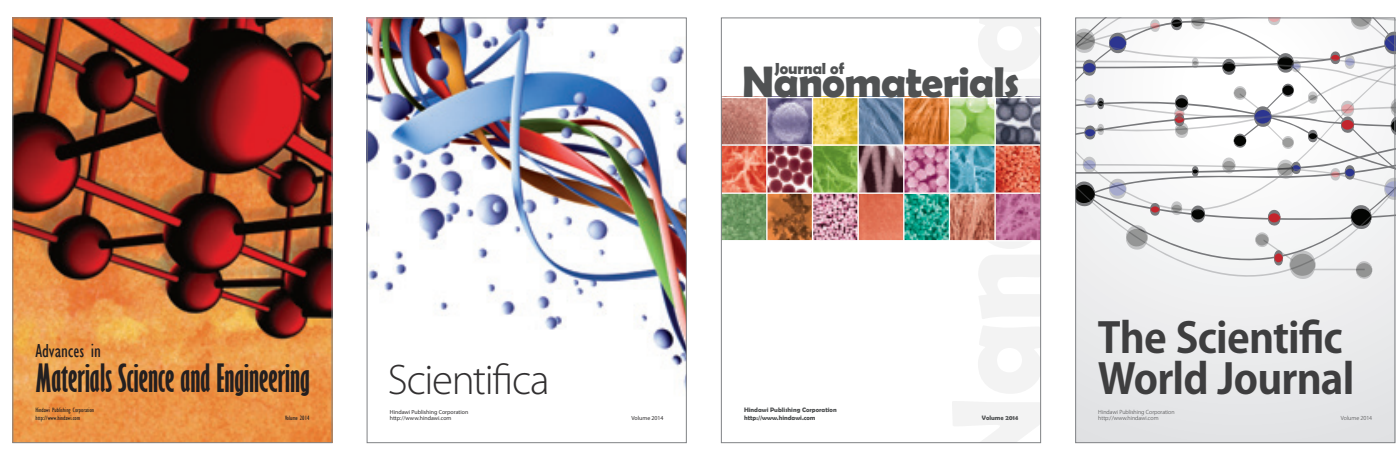

\section{The Scientific World Journal}
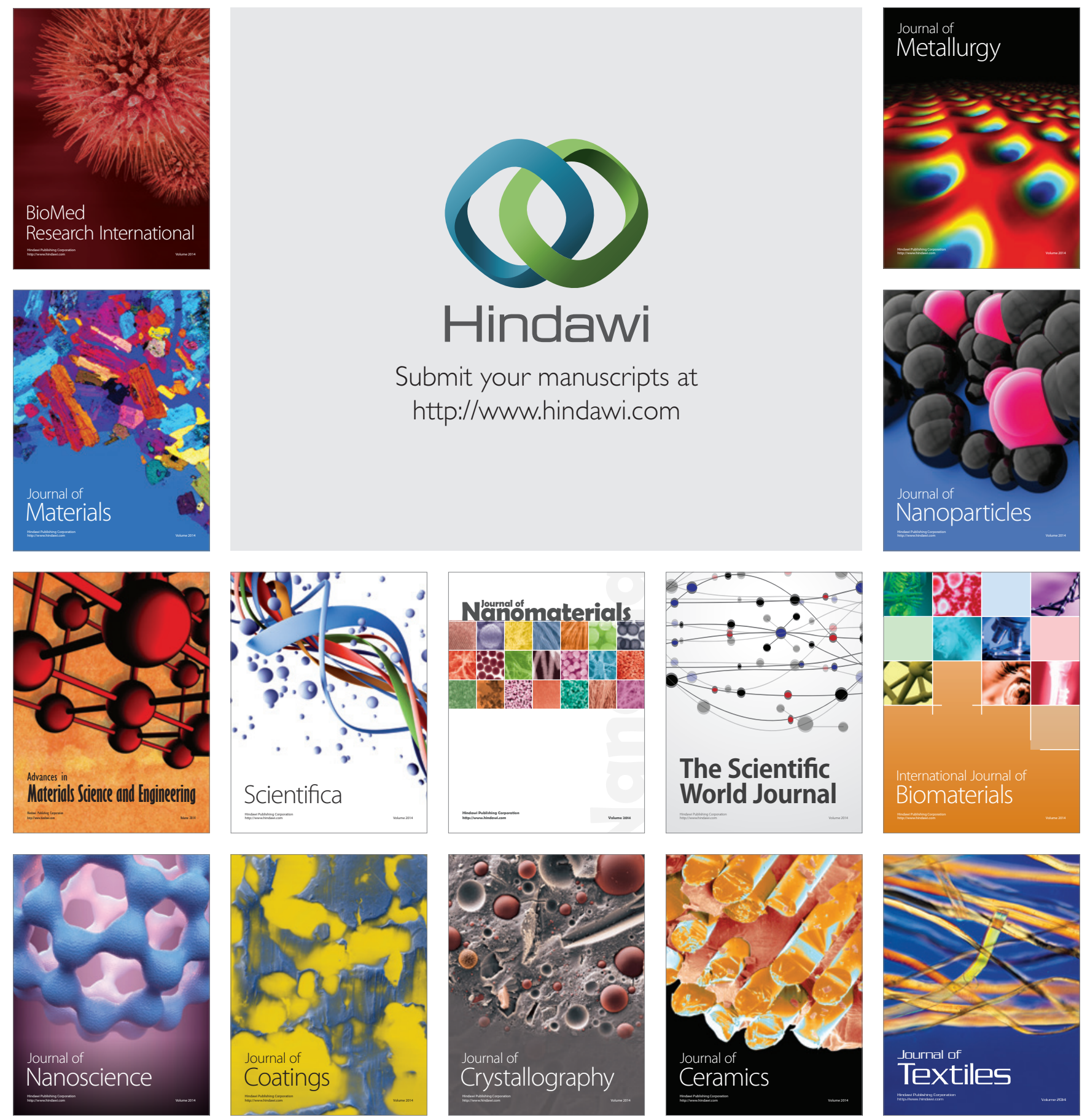